\title{
Childhood Onset of Anti-N-Methyl-D-Aspartate Receptor Encephalitis Without Teratoma Masquerading as a Psychotic Disorder
}

\author{
Tae-Sung Yeum, Jung Lee, Sung-Yeol Park, Yaelim Joen, and Bung-Nyun Kim \\ Division of Child and Adolescent Psychiatry, Department of Psychiatry and Behavioral Science, Seoul National University College of Medicine, \\ Seoul, Korea
}

\begin{abstract}
Many neurologic disorders manifest as psychiatric symptoms. Anti-N-Methyl-D-Aspartate (NMDA) receptor encephalitis is an autoimmune disease of the brain characterized by numerous neurological and psychiatric features. Despite being rare, its prevalence is rapidly increasing and early management is critical in ensuring successful and sustainable recovery. Therefore, the illness should be considered as a differential diagnosis when clinically assessing patients. This report presents a case of a female child who was hospitalized for acute psychiatric manifestations, which was later confirmed as anti-NMDA receptor encephalitis. She recovered relatively successfully after combined neurological and psychiatric treatment. This report provides information on the clinical course of early onset anti-NMDA receptor encephalitis, including treatment strategy and prognosis.
\end{abstract}

Key Words: Anti-N-methyl-D-aspartate receptor encephalitis; N-methyl-D-aspartate receptors; Rituximab.

Received: December 14, 2018 / Revision: March 12, 2019 / Accepted: March 26, 2019

Address for correspondence: Bung-Nyun Kim, Division of Child and Adolescent Psychiatry, Department of Psychiatry and Behavioral Science, Seoul National University College of Medicine, 101 Daehak-ro, Jongno-gu, Seoul 03080, Korea

Tel: +82-2-2072-3647, Fax: +82-2-747-5774, E-mail: kbn1@snu.ac.kr

\section{INTRODUCTION}

Anti-N-Methyl-D-Aspartate (NMDA) receptor encephalitis is a form of inflammation in the brain caused by immune dysfunction. Many efforts have been made to better understand and identify the symptoms of this disease since it was first described in 2007 [1]. The disease presents as a wide range of symptoms of varying severity. Approximately $80 \%$ of patients are female, and in those older than 18 years of age, 50\% have an underlying tumor, which is usually an ovarian teratoma [2]. In children, the first symptoms to be recognized are often non-psychiatric [3].

Although the pathophysiology of the disease is still controversial, researchers and clinicians generally agree that autoantibodies generated by a teratoma or unknown autoimmune mechanism mediate the reaction by targeting NMDA receptors in the brain. This hypothesis of immune-mediated neuronal dysfunction is supported by the fact that the encephalitis is somewhat reversible, irrespective of symptom duration [3]. In addition, absolute levels of NMDA receptor antibodies are higher in the serum than in the cerebrospinal fluid

This is an Open Access article distributed under the terms of the Creative Commons Attribution Non-Commercial License (https://creativecommons.org/licenses/by-nc/4.0) which permits unrestricted non-commercial use, distribution, and reproduction in any medium, provided the original work is properly cited.
(CSF) of patients, suggesting that the antigenic stimulus begins in the peripheral nervous system rather than in the central nervous system [4].

Here, we present the case of a young female who was diagnosed with anti-NMDA receptor encephalitis following 2 months of intensive treatment in a psychiatric inpatient unit, which initially presented as acute psychotic symptoms and bizarre behaviors.

\section{CASE REPORT}

A 9-year-old female was admitted to a closed psychiatric ward for evaluation and treatment of acute behavioral problems. She was born to healthy non-consanguineous parents with a planned pregnancy, and had no perinatal problems. Her neonatal period was unremarkable, including no history of seizure events. She reached regular developmental milestones, except for a delay of several months with respect to language and motor development, which she quickly compensated for. She did not experience separation or stranger anxiety. There was no family history of psychiatric, neurological, metabolic, or genetic disorders.

After a few stressful events related to her academic work, her symptoms began in the form of somatic complaints, such 
as abdominal pain, headache, and eye discomfort. The patient refused to go to school and experienced mild sleep disturbances such as fragmented sleep and early awakening. A week after the first symptoms began, she abruptly developed severe symptoms manifesting as psychotic behavior and cognitive disturbance. The patient suddenly ceased all verbal expression, and occasionally made strange sounds as if she were swimming in water. At night, she did not sleep and cried while displaying signs of irritability and agitation, such as pulling her own hair. The parents attempted to communicate with her about these behaviors; however, she was unable to explain herself logically or provide adequate responses to their questions. A neurological abnormality of mild bilateral stiffness in her upper extremities was also noted.

Brain computed tomography was performed in the emergency room (ER) to rule out any neurological condition such as a vascular disease or tumor. There were no abnormalities in her laboratory examinations; therefore, the patient was referred to a psychiatric department with provisional diagnosis of psychotic disorder not otherwise specified. Her severe condition required intensive treatment and assessment; however, her parents refused to hospitalize her. Thus, she was discharged from the ER with a prescription of risperidone $(1 \mathrm{mg}$ daily), paroxetine (10 $\mathrm{mg}$ daily), and benztropine ( $0.5 \mathrm{mg}$ daily). Continuous medication failed to improve her symptoms, and her psychotic symptoms worsened. She reported both auditory and visual hallucinations. Five days later, she visited the ER again and was hospitalized in the child and adolescent psychiatric inpatient unit for thorough assessment and treatment.

Atypical antipsychotics were initiated to reduce her irritability and psychotic behavior, with a provisional diagnosis of early-onset schizophrenia. Risperidone $0.5 \mathrm{mg}$ was administered daily and titrated up to $0.5-2 \mathrm{mg}$ b.i.d., but the patient experienced acute dystonic reaction. As such, olanzapine was administered as a substitute. Olanzapine $1.25 \mathrm{mg}$ was administered and titrated up to a tolerable dose, which was 2.5-12.5 $\mathrm{mg}$ b.i.d. The treatment slightly improved her irritability and sleep disturbance, but her bizarre behavior continued, and communicating with her remained difficult. There were no clear neurological abnormalities, although she was disoriented in time, place, and person.

Steady antipsychotic treatment did not appear to have an effect; therefore, a neurology consultation was arranged to identify possible neurological causes. A faculty member of the neurology department suggested performing a series of neurologic examinations to rule out organic causes. Ten days after her initial admission, the patient underwent brain magnetic resonance imaging (MRI); however, no abnormalities in the parenchyma or CSF spaces were detected. Medical staff planned to administer electroencephalographic (EEG) recording followed by a spinal tap; however, both tests were postponed because the patient was unable to cooperate for the required procedures to be performed. Autoimmune encephalitis was suggested as a possible diagnosis, as she was too young and the disease progressed too rapidly to be considered early-onset schizophrenia, and she did not respond well to standard antipsychotic treatment. On the 34th day of admission, she underwent an autoimmune antibodies titer, which revealed antibodies to NMDA receptors present in her serum (1:80 titer).

She was treated with methylprednisolone $(25 \mathrm{mg}$ b.i.d. for seven days), intravenous immunoglobulin (10 g daily for five days), and rituximab (400 mg per cycle). Pelvic MRI performed on the 60th day of admission revealed no signs of ovarian teratoma. Meanwhile, $2.5 \mathrm{mg}$ of olanzapine and 0.5 $\mathrm{mg}$ of lorazepam were concurrently administered to manage her residual behavioral symptoms and anxiety, which eventually decreased and ceased by the time of discharge.

Her mental status and behavioral symptoms began to improve as treatment for the encephalitis was initiated. She was discharged on the 107th day of admission following a third cycle of intravenous rituximab treatment. An antibody titer test performed 1 week before discharge reported a weak positive result. At every follow-up, her impulsivity and negativity were found to be gradually improving, and she began to regain her academic abilities. The fifth (and final) cycle of rituximab treatment was administered 63 days after discharge. A follow-up serum autoimmune antibodies titer test was conducted 5 months after the first test. It was negative for anti-NMDA receptor antibody, highlighting the efficacy of her treatment.

Table 1 shows the results of neurocognitive testing performed 4 months after discharge. She communicated easily with the tester and her answers to questions were mostly appropriate during the test. However, the report also noted that she had difficulty performing tests that required verbal expression. Her full-scale Intelligence Quotient was shown to be 76, which is categorized as borderline intellectually functioning. This result was considered to reflect substantial progress, as she had been unable to perform the basic tests or answer simple questions during the acute stages of her disease. The results of the childhood autism rating scale and Asperger syndrome diagnostic scale were normal, and the social maturity index was age-appropriate. Her cognitive function was not considered to have fully recovered compared to her baseline status.

An Advanced Test of Attention was administered to assess symptoms of Attention-Deficit/Hyperactivity Disorder (ADHD) [5]. The patient showed one of four total T-scores 
Table 1. Neurocognitive status of the patient shown by the results of the Korean-Wechsler Intelligence Scale for Children (4th ed)

\begin{tabular}{|c|c|c|c|c|}
\hline Composite & Score & Percentile & Subtest sc & \\
\hline \multirow[t]{5}{*}{ Verbal comprehension } & 90 & 25.2 & Similarities & 9 \\
\hline & & & Vocabulary & 8 \\
\hline & & & Comprehension & 8 \\
\hline & & & (Information) & (10) \\
\hline & & & (Word reasoning) & $(-)$ \\
\hline \multirow[t]{4}{*}{ Perceptual reasoning } & 82 & 12.0 & Block design & 8 \\
\hline & & & Picture concepts & 4 \\
\hline & & & Matrix reasoning & 10 \\
\hline & & & (Picture completion) & (6) \\
\hline \multirow[t]{3}{*}{ Working memory } & 79 & 8.4 & Digit span & 8 \\
\hline & & & Letter-number sequencing & 5 \\
\hline & & & (Arithmetic) & (7) \\
\hline \multirow[t]{3}{*}{ Processing speed } & 74 & 4.0 & Coding & 7 \\
\hline & & & Symbol search & 4 \\
\hline & & & (Cancellation) & $(-)$ \\
\hline Full scale intelligence quotient & 76 & 5.7 & & \\
\hline
\end{tabular}

The test was performed 4 months after discharge and 8 months after the first onset of symptoms
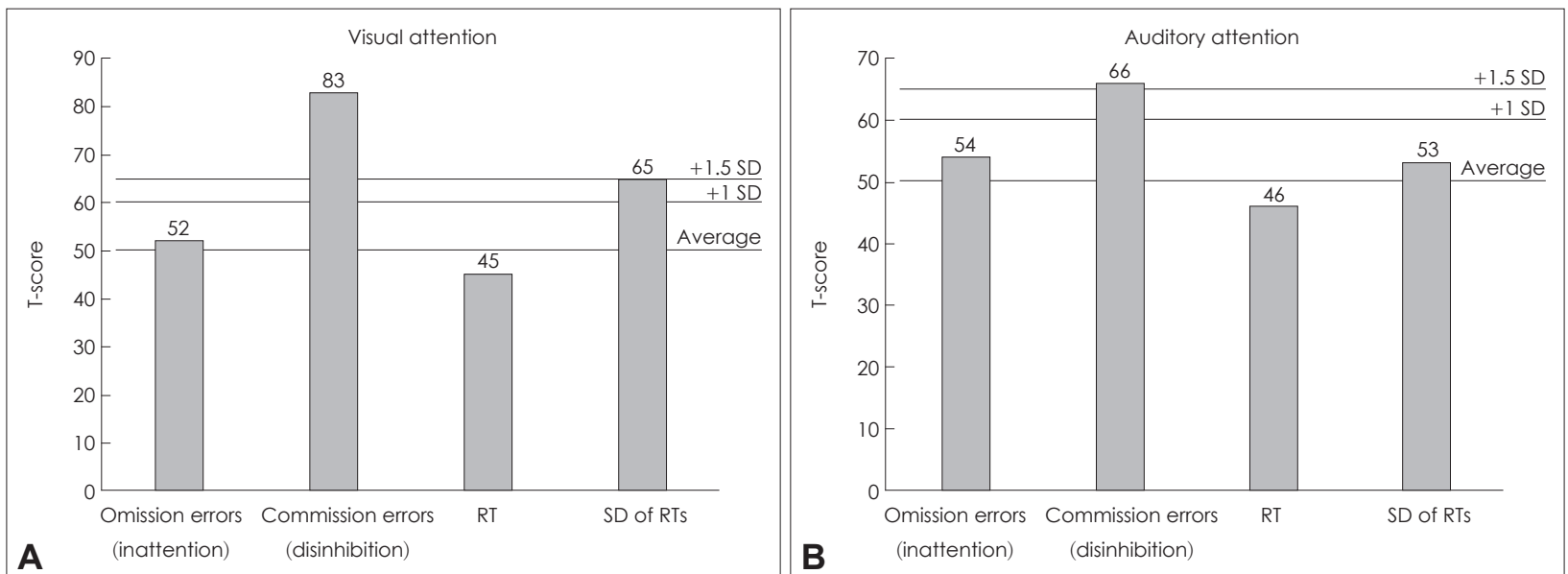

Fig. 1. Result of the Advanced Test of Attention for the patient, performed 4 months after discharge and 8 months after the first onset of symptoms. T-score graphs of the visual (A) and auditory (B) tests. RT: reaction time, SD: standard deviation.

exceeding 65 (over 1.5 standard deviations from the mean), placing the performance of the patient within a range typical of ADHD in both visual and auditory attention tests (Fig. 1). She was especially impaired in the section on "commission errors," indicating impulsivity and disinhibition.

Approximately 1 year after discharge, the patient is clinically well, and her mother has reported that she has nearly returned to a functional level similar to that before the onset of her disease.

\section{DISCUSSION}

Anti-NMDA receptor encephalitis appears to have discrete and often predictable illness phases, showing a viral pro- drome followed by neuropsychiatric symptoms, neurological complications, and prolonged deficits. These stages can help facilitate earlier diagnosis of the syndrome [2]. Herein, we describe a young female who initially presented with a psychotic disorder free of any neurological symptoms, but was subsequently diagnosed with anti-NMDA receptor encephalitis.

In this case, anti-NMDA receptor encephalitis was not an evident differential diagnosis. Illness onset before the age of 12 years is relatively rare, and psychiatric symptoms are usually preceded by prodromal symptoms or neurological impairments [6]. Retrospectively, it is possible that the somatic complaints that she described were initial evidence of her illness, but these nonspecific and mild symptoms remained ambiguous and difficult to detect. Nonetheless, clinicians should 
consider other types of neurological conditions, in addition to anti-NMDA receptor encephalitis, if the clinical course of a psychiatric patient is atypical or the illness presents at a very young age.

Although most patients with anti-NMDA receptor encephalitis show neurological symptoms, pure psychiatric forms of the disease mimicking schizophrenia have been described in previous studies [7,8]. It is therefore important to clinically query anti-NMDA receptor encephalitis in pediatric patients with an atypical course of psychotic and behavioral symptoms with no family history of other psychiatric disorders.

According to a previous report on anti-NMDA receptor encephalitis, 49 out of 58 female patients had ovarian teratoma [9]. However, the number of patients with ovarian teratoma was significantly lower in patients under the age of 18 years [3]. This suggests that an unspecified immunological mechanism other than a teratoma plays an important role in the development of encephalitis in young female patients, as in this case. Other neoplasms, such as lung or breast cancer in NMDA encephalitis were also reported, but the incidence was not considered sufficiently high to warrant further evaluation [3].

Diagnosis of anti-NMDA receptor encephalitis can be made by confirming the presence of antibodies through laboratory tests. Gresa-Arribas et al. [10] reported that detection of antiNMDA receptor antibodies is more sensitive in CSF than in serum. In our case, a positive serum antibody titer contributed to the diagnosis, despite the fact that CSF results were not obtained owing to the child's irritability and uncooperativeness. This often creates a clinical challenge, as patients with the condition often have behavioral problems that make it difficult to conduct CSF analysis.

Measuring antibodies is important not only for the diagnosis of anti-NMDA receptor encephalitis, but also to establish the etiology and prognosis of the condition. A previous study noted that high titers of antibodies in serum and CSF were more likely to reflect an underlying tumor or have worse outcomes compared to those with low titers [10]. It was also mentioned that patients with good outcomes show more rapid and larger decreases in CSF titers than those with poor outcomes. This suggests that favorable outcomes can be expected for our patient, who showed a negative result in the follow-up serum antibodies titer.

Although patients can show abnormalities such as hyperintensities on brain MRI [9], the neuroimaging findings were normal in our case. In addition, there have been reports of abnormal EEG findings, such as peculiar and recurrent paroxysmal nonepileptic rhythmic theta-delta patterns [11], or a pattern of extreme delta brush [12], neither of which were observed in this patient. These MRI and EEG abnormalities may be features of anti-NMDA receptor encephalitis that lack the sensitivity and specificity to warrant their use as diagnostic tests [13]. According to a previous case series report, $89 \%$ of patients with anti-NMDA receptor encephalitis under 12 years old had abnormal EEG recordings. In addition, $55 \%$ had abnormal findings on brain MRI, such as contrast enhancement or abnormal diffusion weighted images [6]. The inconsistency of our patient's results with these emphasized that it is an atypical case.

The standard first-line management for anti-NMDA receptor encephalitis consists of removing the causative tumor, which was not required in this case. Data on the targeted management of psychiatric symptoms in autoimmune encephalitis are currently limited. The use of multiple classes of psychotropic medications is detailed in a number of case reports, but the most profound improvements in psychiatric and behavioral symptoms occur when the antibody response is suppressed or reversed [14]. Analysis of anti-NMDA receptor antibodies in patients with encephalitis is important to identify an autoimmune etiology and promptly initiate immunotherapy to minimize adverse sequelae. Rituximab should be considered in patients without teratomas, or in patients who do not improve with other immunosuppressive treatments [15]. This patient was treated sequentially with different forms of immunotherapy. Among them, rituximab was the only one that elicited a clear response. Owing to her behavioral problems, it was thought that the patient still required a certain amount of psychotropic medication even after immunotherapy commenced. As such, her psychotropic medications were only slowly tapered off over the course of her outpatient visits.

It is noteworthy that the patient's remaining symptoms of inattention and impulsivity mimicked those of ADHD following treatment with immunotherapy. It is considered highly unlikely that she already had the clinical manifestation of ADHD, because the patient had been attaining excellent grades at school, and her parents and school records report no previous symptoms of inattention, hyperactivity, or impulsivity. Previous studies reporting that there was a significant association between anti-NMDA receptor encephalitis and symptoms of ADHD also support this finding [2,16,17]. It is not known whether these symptoms would improve in response to traditional ADHD treatments, such as administration of psychostimulants, because the symptoms stemmed from the encephalitis. Further research will be needed to elucidate this.

Anti-NMDA receptor encephalitis can induce substantial and persistent cognitive impairments, with motor, language, and memory deficits, reflective of disruptions to synaptic processes in the hippocampus and its target areas [18,19]. A remarkable observation is that early immunotherapy is associ- 
ated with significantly better cognitive outcomes [20]. This demonstrates the importance of making a correct diagnosis early and commencing treatment as soon as possible.

In conclusion, acute psychotic symptoms or unexplained behavioral problems in young female patients may be the only initial manifestation of this rare disease of anti-NMDA receptor encephalitis. Disease suspicion and careful historytaking of the illness onset is crucial. The prompt initiation of immunotherapy could alleviate the symptoms, while minimizing the complications linked to persistent cognitive impairment.

\section{Acknowledgments}

This work was supported by a National Research Foundation of Korea (NRF) grant funded by the Korean Government (MSIP; No. 2015M3C7A1028926 to B.-N.K).

\section{Conflicts of Interest}

The authors have no potential conflicts of interest to disclose.

\section{REFERENCES}

1) Dalmau J, Tüzün E, Wu HY, Masjuan J, Rossi JE, Voloschin A, et al. Paraneoplastic anti-N-methyl-D-aspartate receptor encephalitis associated with ovarian teratoma. Ann Neurol 2007;61:25-36.

2) Kayser M, Dalmau J. Anti-NMDA receptor encephalitis in psychiatry. Curr Psychiatry Rev 2011;7:189-193.

3) Dalmau J, Lancaster E, Martinez-Hernandez E, Rosenfeld MR, Balice-Gordon R. Clinical experience and laboratory investigations in patients with anti-NMDAR encephalitis. Lancet Neurol 2011;10: 63-74.

4) Irani SR, Bera K, Waters P, Zuliani L, Maxwell S, Zandi MS, et al. $\mathrm{N}$-methyl-D-aspartate antibody encephalitis: temporal progression of clinical and paraclinical observations in a predominantly nonparaneoplastic disorder of both sexes. Brain 2010;133:1655-1667.

5) Shin MS, Cho S, Chun SY, Hong KE. A study of the development and standardization of ADHD diagnostic system. J Korean Acad Child Adolesc Psychiatry 2000;11:91-99.

6) Armangue T, Titulaer MJ, Málaga I, Bataller L, Gabilondo I, Graus F, et al. Pediatric anti-N-methyl-D-aspartate receptor encephalitisclinical analysis and novel findings in a series of 20 patients. J Pediatr 2013;162:850-856.e2.

7) Kayser MS, Titulaer MJ, Gresa-Arribas N, Dalmau J. Frequency and characteristics of isolated psychiatric episodes in anti-N-meth- yl-d-aspartate receptor encephalitis. JAMA Neurol 2013;70:11331139.

8) Titulaer MJ. Anti-NMDA receptor encephalitis mimicking schizophrenia: diagnosis and treatment. Eur Neuropsychopharmacol 2016; 2:S134-S135.

9) Dalmau J, Gleichman AJ, Hughes EG, Rossi JE, Peng X, Lai M, et al. Anti-NMDA-receptor encephalitis: case series and analysis of the effects of antibodies. Lancet Neurol 2008;7:1091-1098.

10) Gresa-Arribas N, Titulaer MJ, Torrents A, Aguilar E, McCracken L, Leypoldt F, et al. Diagnosis and significance of antibody titers in anti-NMDA receptor encephalitis, a retrospective study. Lancet Neurol 2014;13:167-177.

11) Nosadini M, Boniver C, Zuliani L, De Palma L, Cainelli E, Battistella PA, et al. Longitudinal electroencephalographic (EEG) findings in pediatric anti-N-methyl-D-aspartate (anti-NMDA) receptor encephalitis: the Padua experience. J Child Neurol 2015;30:238-245.

12) Schmitt SE, Pargeon K, Frechette ES, Hirsch LJ, Dalmau J, Friedman D. Extreme delta brush: a unique EEG pattern in adults with anti-NMDA receptor encephalitis. Neurology 2012;79:1094-1100.

13) Gable MS, Sheriff H, Dalmau J, Tilley DH, Glaser CA. The frequency of autoimmune N-methyl-D-aspartate receptor encephalitis surpasses that of individual viral etiologies in young individuals enrolled in the California Encephalitis Project. Clin Infect Dis 2012;54:899904.

14) Chapman MR, Vause HE. Anti-NMDA receptor encephalitis: diagnosis, psychiatric presentation, and treatment. Am J Psychiatry 2011; 168:245-251.

15) Ishiura $H$, Matsuda $S$, Higashihara $M$, Hasegawa $M$, Hida $A$, Hanajima R, et al. Response of anti-NMDA receptor encephalitis without tumor to immunotherapy including rituximab. Neurology 2008; 71:1921-1923.

16) Houtrow AJ, Bhandal M, Pratini NR, Davidson L, Neufeld JA. The rehabilitation of children with anti-N-methyl-D-aspartate-receptor encephalitis: a case series. Am J Phys Med Rehabil 2012;91:435-441.

17) Reid DK, Clardy SL. Anti-NMDA-receptor encephalitis: unusual presentation of an uncommon condition. J Neurol Neurosurg Psychiatry 2013;84:69-70.

18) Hughes EG, Peng X, Gleichman AJ, Lai M, Zhou L, Tsou R, et al. Cellular and synaptic mechanisms of anti-NMDA receptor encephalitis. J Neurosci 2010;30:5866-5875.

19) Hinkle CD, Porter JN, Waldron EJ, Klein H, Tranel D, Heffelfinger A. Neuropsychological characterization of three adolescent females with anti-NMDA receptor encephalitis in the acute, post-acute, and chronic phases: an inter-institutional case series. Clin Neuropsychol 2017;31:268-288.

20) Finke C, Kopp UA, Prüss H, Dalmau J, Wandinger KP, Ploner CJ. Cognitive deficits following anti-NMDA receptor encephalitis. J Neurol Neurosurg Psychiatry 2012;83:195-198. 